\title{
The Interplay between Geometry and Function in the Comprehension of Over, Under, Above, and Below
}

\author{
Kenny R. Coventry, Mercè Prat-Sala, and Lynn Richards
}

Centre for Thinking and Language, Department of Psychology, University of Plymouth, Plymouth, United Kingdom

\begin{abstract}
Three experiments are reported which examined the role of geometry and functional relations in the comprehension of the spatial prepositions over, under, above, and below. The tasks used consisted of rating how appropriate sentences (containing one of these prepositions) were to describe a series of pictures. For example, the pictures comprised a person holding an object with the function of protection from falling objects (e.g., an umbrella). Each picture was depicted with the object shown as fulfilling its function or not at different geometric positions. The results of Experiment 1 show a significant effect of functional relations on the ratings given. However, while over/under were very sensitive to functional relations, above/below were more influenced by geometric relations. The second experiment replicates these effects with objects depicting noncanonical functions (e.g., a suitcase sheltering someone from rain). Experiment 3 manipulated frame of reference and found evidence for conflict of frames of reference effects on the rating of above/below, but not in the same way for over/under. Conversely the ratings of over/under were found to be affected by functionality while those for above/below were not. These results indicate for the first time that spatial prepositions are differentially influenced by geometric and functional relations. () 2001 Academic Press
\end{abstract}

Key Words: spatial prepositions; function; geometry; reference frames.

Being able to find objects in the world is one of the most basic survival skills required by any living organism. Similarly, being able to describe where objects are and being able to find objects based on simple locative descriptions can be regarded as basic skills for a competent speaker of a language. The use of an expression involving a spatial preposition in English conveys to a hearer where one object (figure) is located in relation to a reference object (ground). For example, in "the pear is in the bowl," the pear is understood to be located with reference to the bowl in the region denoted by the preposition in. Understanding the meaning of spatial prepositions is of particular importance in semantics as they are among the set of closed class

This research was supported by a grant from the Economic and Social Research Council (Grant R000222211) to the first author. We thank two anonymous referees for helpful comments on the first version of the manuscript. We also thank Simon Venn for drawing the pictures.

Address correspondence and reprint requests to Kenny R. Coventry, Centre for Thinking and Language, Department of Psychology, Faculty of Human Sciences, University of Plymouth, Drake Circus, Plymouth PL4 8AA, UK. E-mail: K.Coventry@plymouth.ac.uk. terms which are generally regarded as having the role of acting as organizing structure for further conceptual material (Talmy, 1983; Lakoff, 1987). Furthermore, spatial prepositions have the virtue of relating to measurable characteristics of the world being described (Regier, 1996). Therefore it should be possible to specify the components in a visual scene which are the predictors of comprehension for individual spatial terms.

Despite the relatively small number of spatial prepositions denoting place in English (around only 80-100; Landau \& Jackendoff, 1993), it turns out to be difficult to tie down the conditions under which such terms are used. Traditionally, geometric constructs have been invoked to underpin their lexical entries (e.g., Herskovits, 1986; Logan \& Sadler, 1996). For example, in the sentence, "The pear is in the bowl," the figure (the pear) is located in the region described by the prepositional phrase in the bowl, with the spatial relation expressed by in corresponding to "contained interior to the reference object." However, there is not a direct mapping between spatial relations and prepositional usage. For instance, in is appropriate to describe the relationship between the pear and 
the bowl in Fig. 1a but over would be the most appropriate term in Fig. 1b although the geometric relations between figure and ground are identical. Clearly, while geometry is important in the use and comprehension of spatial prepositions, other extrageometric variables need to be invoked in order to account for use and comprehension. For example the expression, the man is at the piano, implies that the man is playing the piano, not just that he is in close proximity to it. To give another example, while the position of the umbrella is undoubtedly important in the comprehension of the umbrella is over the man, it is likely that the function of the figure (to protect someone from rain) is also important in the comprehension of the expression. The main area of investigation in the present paper is the relative extent to which geometric relations versus extrageometric relations are predictors of the comprehension of spatial prepositions, focussing on over, under, above, and below. Furthermore, we test the intuition that such extrageometric information may be more important for some spatial prepositions (e.g., over and under) than for others (e.g., above and below). Before focusing on these prepositions in more detail, we briefly review evidence for the importance of extrageometric variables in the use and comprehension of spatial prepositions.

\section{Evidence for the Importance of Extrageometric}

Factors in the Use and Comprehension of

\section{Spatial Prepositions}

Recently a number of researchers has argued that extrageometric factors play an important role in the use and comprehension of spatial preposi-

(a)

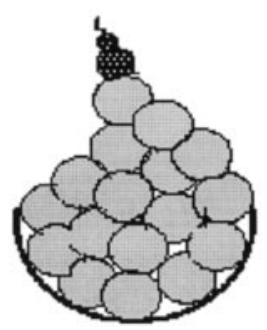

(b)

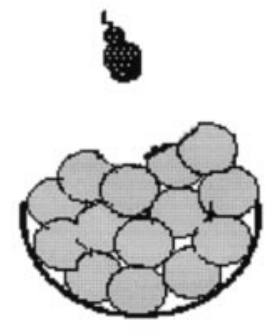

FIG. 1. (a) The pear is in the bowl. (b) *The pear is in the bowl. tions. In particular, functional relations have been postulated as key components underlying the meaning of the spatial prepositions in, on, and at (Coventry, 1992, 1998; Coventry, Carmichael, \& Garrod, 1994; Garrod \& Sanford, 1989; Garrod, Ferrier, \& Campbell, 1999; Talmy, 1988; Vandeloise, 1994). Functional relations have to do with how objects interact with each other and what the functions of objects are. For example, with in, Garrod and Sanford (1989) and Coventry $(1992,1998)$ propose that the lexical entry is

in: functional containment-in is appropriate if the ground is conceived of as fulfilling its containment function.

Whether or not in is appropriate depends on a number of factors which determine whether the container is fulfilling its function. These include movement over time where the figure remains in the same position relative to the container, or where the container is sealed, thus blocking movement of the figure beyond the rim of the container, allowing constraint of location over time.

Empirical evidence for the importance of this functional analysis has been forthcoming for topological prepositions. For example, Coventry $(1992,1998)$ found that contiguity of movement of the figure with the ground significantly increased the use and rating of in while independent movement of the figure decreased ratings when the figure was positioned on top of other objects above the rim of a container as compared with static scenes where the geometry was the same. Similarly, tilting the container reduces the use of in if it looks like the figure may fall out. Garrod, et al. (1999) have also shown that judgements of the ratings of in and on correlate with independent judgements of movement (functional control).

In addition to the effects of movement, Coventry, et al. (1994) provide preliminary evidence that use and ratings of prepositions can be influenced by grounds which have the same function but usually contain different objects. When they compared static scenes involving a jug and a bowl, in was used significantly more with the bowl as ground than with the jug as ground when the figure was a solid on top of a 
pile of other solids above the rim of the container. Furthermore, adding liquid to the jug was found to further decrease the use (and ratings) of in, but it made no difference in the case of the bowl. Thus the addition of water appears to make the object-specific function of the jug (to contain liquids) more salient, further reducing the appropriateness of the container as a container of solids. Additionally, Coventry et al. (1994) found that labeling the same object a dish versus a plate influences prepositional usage, indicating that different nouns suggest different objectspecific properties.

\section{The Relative Importance of Geometric and}

Extrageometric Variables in the Use and

\section{Comprehension of Spatial Prepositions}

While the importance of extrageometric relations in the use and comprehension of a range of spatial prepositions has been established, there are two central issues which the studies reported below aim to address. First, although both geometric and extrageometric factors are clearly implicated in the use and comprehension of spatial terms, it needs to be established whether geometric variables dominate extrageometric variables or whether these variables are equally important in the use and comprehension of spatial terms. On the one hand, Coventry (1998) and Garrod and Sanford (1989) have argued for the centrality of extrageometric variables and trace the effect of these variables back to the lexical entries for prepositions. On the other hand, Landau and Munnich (1998) have suggested an alternative interpretation of the empirical findings outlined above. While recognizing that extrageometric variables are important, they argue that these kinds of effects are essentially added onto the geometry to modify the geometric regions appropriate. Furthermore, Landau and Munnich argue that these geometric regions are well specified. This view would appear to be in line with that of Herskovits (1986), who has outlined a set of pragmatic principles (termed near principles) which bend and stretch the geometric constraints embodied in the lexicon under some circumstances. For example, Herskovits has argued that in is appropriate in Fig. 1a to describe the location of the pear in relation to the bowl as a result of the principle of tolerance, which allows in to be appropriate as the figure is part of a group of other objects, some of which meet the containment condition. In this way a pragmatic principle allows an extension of the geometric containment region applicable. The studies reported test directly which of these views is most plausible.

The second main issue we wish to deal with is the notion that prepositions may be differentially influenced by extrageometric variables. In the preposition literature, extrageometric constraints have been flagged for some prepositions but not others. For example, in linguistic accounts it is well recognized that there are uses of in and at which involve a functional component (Herskovits, 1986; Aurnague, 1995). On the other hand, prepositions like above have been regarded as being more simply geometric, specifying a higher than relation in Euclidean space (e.g., Bennett, 1975). In particular, we examine the differential effects of geometry and extrageometric variables on the comprehension of over, above, under, and below.

\section{Over, Under, Above, and Below}

The role of geometry underpinning the use and comprehension of projective prepositions such as over, under, above, and below has been the subject of both extensive linguistic analyses (e.g., Bennett, 1975; Brugman, 1988; Lakoff, 1987) and recent empirical study (CarlsonRadvansky \& Irwin, 1993, 1994; CarlsonRadvansky \& Radvansky, 1996; Hayward \& Tarr, 1995; Logan \& Sadler, 1996). Logan and Sadler (1996) found that the prototypical above relationship is at a point higher than directly above the reference object, in line with the center of mass of the reference object. Displacing the figure from the central axis of the reference object was found to reduce the appropriateness of the term (and other prepositions as well). However, these studies involved participants marking points on or rating scenes involving abstract geometric shapes rather than real objects with any relations between them.

The majority of studies examining over, under, above, and below have dealt with the issue of frame of reference for these terms. One can use 
prepositions like above with respect to one (or more) of three basic reference frames (Levinson, 1996). These are the intrinsic (or objectcentered), relative (or viewer-centered/deictic), or absolute (environment-centered or extrinsic) frames. The intrinsic frame locates a figure with reference to the salient features of the ground. For example, the car is behind the house used intrinsically locates the car in relation to the opposite wall from where the salient front of the house is, which is where the back door is. The relative use of the same expression would locate the car directly behind the opposite wall to the wall where the speaker and hearer are standing. The most common absolute use relates to the gravitational plane where terms like over and above are used for positions higher than in the gravitational plane. Now in relation to extrageometric relations, Carlson-Radvansky and Radvansky (1996) have found that the presence of a functional relation between objects to be described influences the choice of reference frame used to describe objects. Imagine a picture of a mail carrier holding a letter standing near and to the left of a mailbox. When the mail carrier was standing facing the mailbox with hand outstretched as if to be posting the letter, then participants had a preference for using intrinsic descriptions (e.g., the mail carrier is in front of the mailbox). In contrast, when the mail carrier was standing with his back to the mailbox, then extrinsic-relative descriptions were preferred (e.g., the mail carrier is to the left of the mailbox). While this provides evidence for the importance of functional relations in the selection of a frame of reference for projective terms, CarlsonRadvansky and Radvansky (1996) did not examine the effects of functional relations on the use of terms within a single frame of reference when functional relations are varied. They also manipulated functional relations by changing the position of the figure, and consequently their research does not address the issue of the relative importance of geometry and functionality for spatial description. The third experiment reported below examines the comprehension of over, under, above and below in cases where frames of reference coincide versus conflict by manipulating both geometry and functional relations. The first two experiments reported manipulate these variables when frames of reference coincide.

The other main issue of interest examined below is the relative extent to which prepositions are influenced by geometric and extrageometric variables. In the preposition literature, although extrageometric effects have been documented, the issue of the relative extent to which terms are influenced by these variables is not considered. Although Carlson-Radvansky, Covey, and Lattanzi (1999) have shown that above can be influenced by functional relations and context, Coventry and Mather (in press) have suggested that over is more influenced by extrageometric factors than above, and that prepositions may cluster into functional and nonfunctional groups. Furthermore, Coventry and Mather link this hypothesis to the extent to which prepositions are polysemous. However, neither team of researchers manipulated geometry and function together with the four prepositions over, under, above, and below in order to adequately test whether prepositions are differentially affected by these variables. The studies below do this for the first time. Our predictions were that over and under should be more influenced by extrageometric variables than above and below, while above and below should be more influenced by geometric manipulations.

In addition to the main manipulations, the experiments also aimed to tease apart the influence of a number of distinct extrageometric variables previously labeled under the same banner of functional relations in the literature. For example, Coventry (1998) on the one hand provides evidence for the importance of the interaction between objects in the ratings and use of in, and on the other hand, Coventry et al. (1994) demonstrate effects which have to do with the usual function of objects (e.g., a jug versus a bowl). The first experiment examines cases in which the objects are shown to be either fulfilling or not fulfilling their canonical functions (protecting functions for one set of materials and containment functions for the other set), while the second experiment examines whether an object without a usual protecting function still illustrates function effects if it is depicted as protecting (i.e, functioning noncanonically) in the context. 


\section{EXPERIMENT 1}

Experiment 1 manipulated geometry and function independently and together in order to assess the relative influence of both factors on the comprehension of over, above, under, and below. Given the myriad of object knowledge effects reported for other prepositions, the experiment used two sets of materials involving two different types of functional relations. The first set involved figures with protecting functions (the function of protection from falling objects), and the second set involved grounds with containment functions and object pairs with relations highlighted by how successfully they were interacting with one another. While function and geometry effects were both expected to be present, it was predicted that function effects should be more in evidence with the prepositions over and under than with above and below. Conversely it was predicted that above and below would be more affected by changes in geometric relations than over and under.

\section{Design}

The experiment was designed to test for the differential effects of function and geometry on participants' ratings of the appropriateness of sentences to describe a series of pictures. The variables manipulated included three levels of geometry and three levels of functionality. The figure in the picture (for material set 1) was positioned canonically directly above the ground (i.e., in the usual position), at an angle of $45^{\circ}$, or at an angle of $90^{\circ}$ to the ground (see Fig. 2). For each level of geometry three levels of functionality were employed. The figure was shown fulfilling its function, the figure was shown not fulfilling its function, or other objects were not present to make the functional relationship relevant (a control). The sentences given to rate were presented in pairs for each picture. The sentences in a pair were identical except for the preposition. For example, a pair of sentences could be The man is under the umbrella and The man is below the umbrella. The predictions were that if geometric relations had an effect on the comprehension of these prepositions, the participants would give the highest ratings to a sen- tence when it described a picture where one of the objects appeared in its canonical position and the lowest ratings when the same sentence described a variant of the picture which contained the same object tilted at $90^{\circ}$. We were also predicting that if functional relations had an effect, participants' ratings for a particular sentence would be higher when it described a picture depicting a functional relation between objects than when the functional relation was not present. Additionally, it was predicted that over and under would be most sensitive to functional relations while above and below should be more sensitive to geometric relations.

\section{Method}

Participants. Thirty-eight undergraduate students from the University of Plymouth participated in this experiment as an extra credit option in a Psychology course. All participants were native speakers of English.

Materials. The materials for the experiment consisted of a total of 144 pictures and were based on two sets of four types of pictures. The first set of pictures involved the use of a figure with the function of protection from falling objects. For these scenes, the figure was rotated. The second set involved the use of a ground with the function of containment. Object pairs with a stronger association with one another in terms of object relationship were used for this set (e.g., a bottle and a glass), and for these scenes the ground was rotated.

Each picture in a set had nine variants (three levels of geometry and three levels of functionality), making a total of 72 pictures. Each picture was printed twice, once with a pair of sentences (e.g., The man is under/below the umbrella) and once with another pair of sentences (e.g., The umbrella is over/above the man) to test all four prepositions.

The first set of pictures depicted a person using an object to protect himself from a falling object/objects (e.g., a man using an umbrella to protect himself from rain). The objects used were an umbrella (protecting a man), a shield (protecting a Viking), a visor (protecting a gardener), and a hard hat (protecting a workman). For each picture there were three levels of geom- 

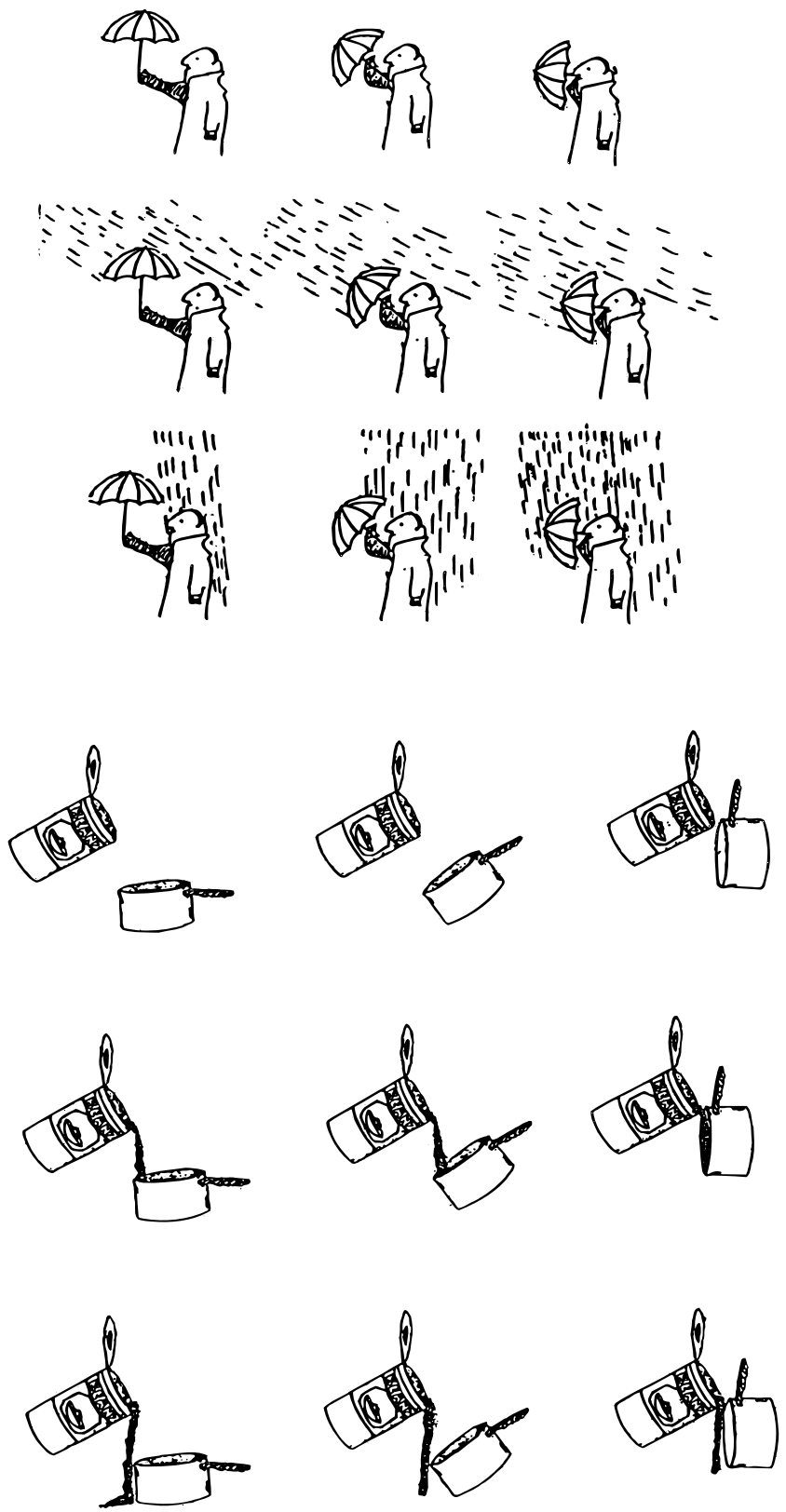

FIG. 2. Examples of materials used in Experiment 1 (also see footnote 1).

etry of the object used by the man to protect himself (in this case the umbrella). The figure in the picture was positioned canonically directly above the ground, at an angle of $45^{\circ}$, or at an angle of $90^{\circ}$ to the ground. Additionally, for each geometric permutation there were three levels of functionality: control, where the object the man tried to protect himself from was absent (e.g., there was no rain); functional, where the object was present (i.e., the rain was falling on the umbrella keeping the man dry); and nonfunctional, where the falling object was present 
but the protecting object did not fulfil its function (i.e., the rain was falling on the man despite the umbrella's presence). See Fig. 2 for an example of all nine levels of picture for one set of materials. ${ }^{1}$

The second set of pictures depicted two related objects (e.g., a can and a pan). One of these objects was always a recipient container. The object sets used were a can and a pan, a bottle and a glass, a chute and a skip, and a tap and a bucket. For each picture there were three levels of geometry of the recipient object (in this case the pan). The ground in the picture was positioned canonically directly below the figure, at an angle of $45^{\circ}$, or at an angle of $90^{\circ}$ to the figure. There were also three levels of functionality: control, where the falling/pouring object was absent (in this case there were no beans in the can); functional, where the falling/pouring object was present (i.e., there were beans falling into the pan); and nonfunctional, where the falling/pouring object was present but did not end up in the recipient object (i.e., there were beans missing the pan and dropping onto the floor). See Fig. 2 for an example of a picture with all nine levels of function/geometry.

\section{Procedure}

Participants were run in groups of 6-10 people. Each participant received the instructions and a booklet containing the materials. The participants were told that each page of the booklet contained a picture and two sentences, and that their task consisted of rating how appropriate each sentence was to describe the picture using a 7-point scale, where 1 meant totally inappropriate and 7 totally appropriate. Participants were free to use any number in the scale. The experimental sessions lasted around $30 \mathrm{~min}$.

The test materials were divided into two parts. One copy of each picture (with one pair of prepositions) appeared in part one, and the other copy of the picture (with the other pair of prepositions) appeared in part two. Half of the participants saw part one first, and the other half saw

\footnotetext{
${ }^{1}$ Further example scenes from all three experiments can be found at the following website: http://psy.plym.ac.uk/ staff/kcoventry/spatpics 1.html.
}

the second part first (though participants were not aware of the two parts). Additionally, the materials were randomized in a stratified fashion. We created nine groups of eight pictures for each part. Each group had one picture from each material set and corresponded to a different level of geometry and functionality. Then, each individual group was randomized before being grouped with another group. Each participant had a different grouping and randomization. The end result of this process meant that the possibility of priming effects was minimized.

\section{Results}

The results for each set of materials are reported separately, and the data are displayed (mean ratings) in the Appendix, Tables A1 and A2. Analyses by materials and by inferior/superior preposition sets (over/above versus under/ below) were also undertaken for this experiment and the following experiments, but they produced results of little interest, so they are not reported here. Where follow-up analyses are reported, Tukey (HSD) tests were used.

Material Set 1. A three-way analysis of variance (fully within) was performed on the rating data. The results of the analysis are displayed in Table 1. The variables were function (e.g., functional, nonfunctional, and control), angle (canonical, $45^{\circ}$, or $90^{\circ}$ ), and preposition set (over/ under versus above/below). The mean ratings are displayed in the Appendix, Table A1.

As expected, a significant main effect of angle was found. The highest ratings were given for the canonical orientation (mean rating $=4.16$ ), followed by $45^{\circ}$ angle (mean $=3.77$ ), and the lowest were given for the $90^{\circ}$ angle pictures $($ mean $=2.27)$. There was also a significant main effect of function, and all three levels differed significantly from one another. The highest ratings were given for the functional scenes (mean $=3.81)$, the next highest for the control scenes (mean $=3.53$ ), and the lowest ratings were given for the nonfunctional scenes (mean $=2.87)$. A significant interaction between function and angle was also present. This is displayed in Fig. 3. The nonfunctional scenes were given significantly lower ratings than the other two levels for all three positions, but the functional scenes 
TABLE 1

Results of Three-Way ANOVA for Experiment 1, Material Set 1

\begin{tabular}{lccc}
\hline \multicolumn{1}{c}{ Source } & df and F value & $\mathrm{MS}_{e}$ & Significance \\
\hline Functionality (F) & $\mathrm{F}(2,72)=82.41$ & 0.62 & $* * *$ \\
Angle (A) & $\mathrm{F}(2,72)=213.22$ & 1.04 & $* * *$ \\
Preposition set (PS) & $\mathrm{F}(1,36)=76.07$ & 1.71 & $* * *$ \\
$(\mathrm{~F}) \times(\mathrm{A})$ & $\mathrm{F}(4,144)=5.36$ & 0.25 & $* *$ \\
$(\mathrm{~F}) \times(\mathrm{PS})$ & $\mathrm{F}(2,72)=34.36$ & 0.50 & $* * *$ \\
$(\mathrm{~A}) \times(\mathrm{PS})$ & $\mathrm{F}(2,72)=122.15$ & 1.00 & $\mathrm{~ns}$ \\
$(\mathrm{~F}) \times(\mathrm{A}) \times(\mathrm{PS})$ & $\mathrm{F}(2,72)=1.19$ & 0.20 & \\
\hline
\end{tabular}

Note. ns, $p>.05$.

$* p<.01$.

$* * p<.001$.

$* * * p<.0001$.

were given significantly higher ratings than the controls only for the $45^{\circ}$ and $90^{\circ}$ angles.

Of most interest in the analyses were the main effect for preposition set and the significant interactions between this variable and angle and function. Overall over/under were given lower ratings than above/below. However, the inter- actions between over/under-above/below and angle (displayed in Fig. 4) and between over/ under-above/below and function (displayed in Fig. 5) provide evidence that these preposition sets behave differently from one another. Over/ under were found to be more affected by the functional manipulation than above/below, while

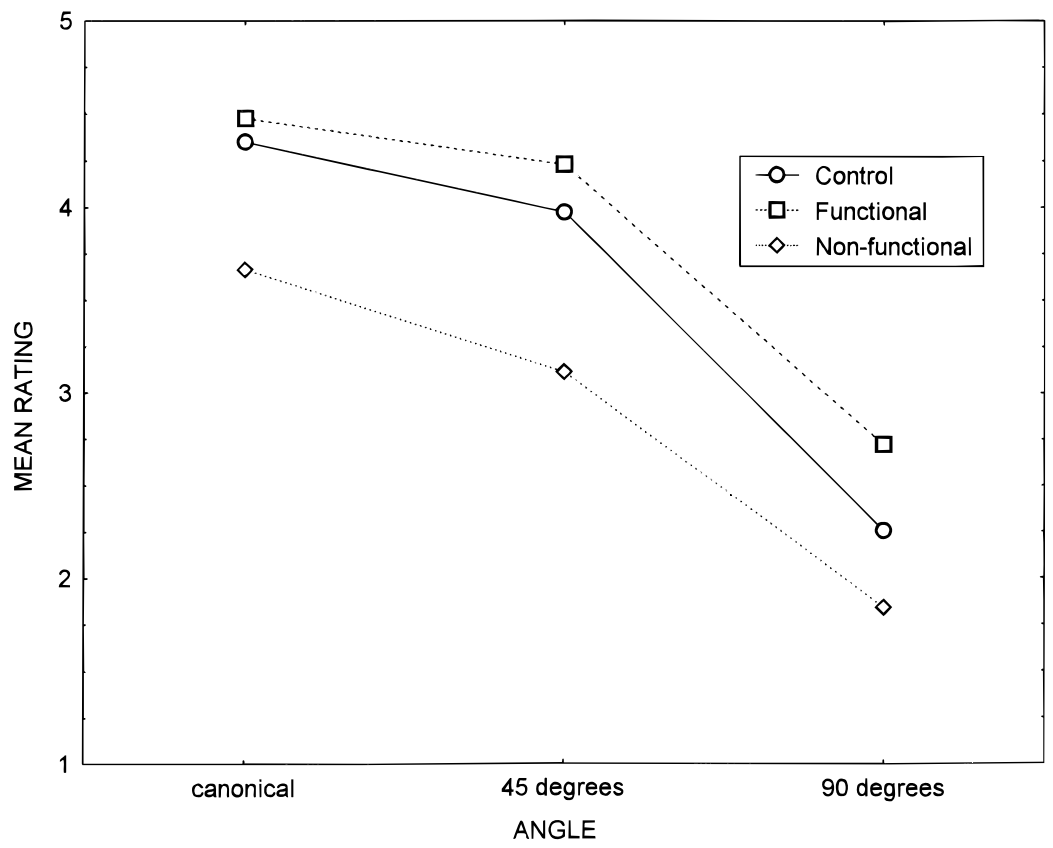

FIG. 3. Interaction between geometry and function for Material Set 1, Experiment 1. 


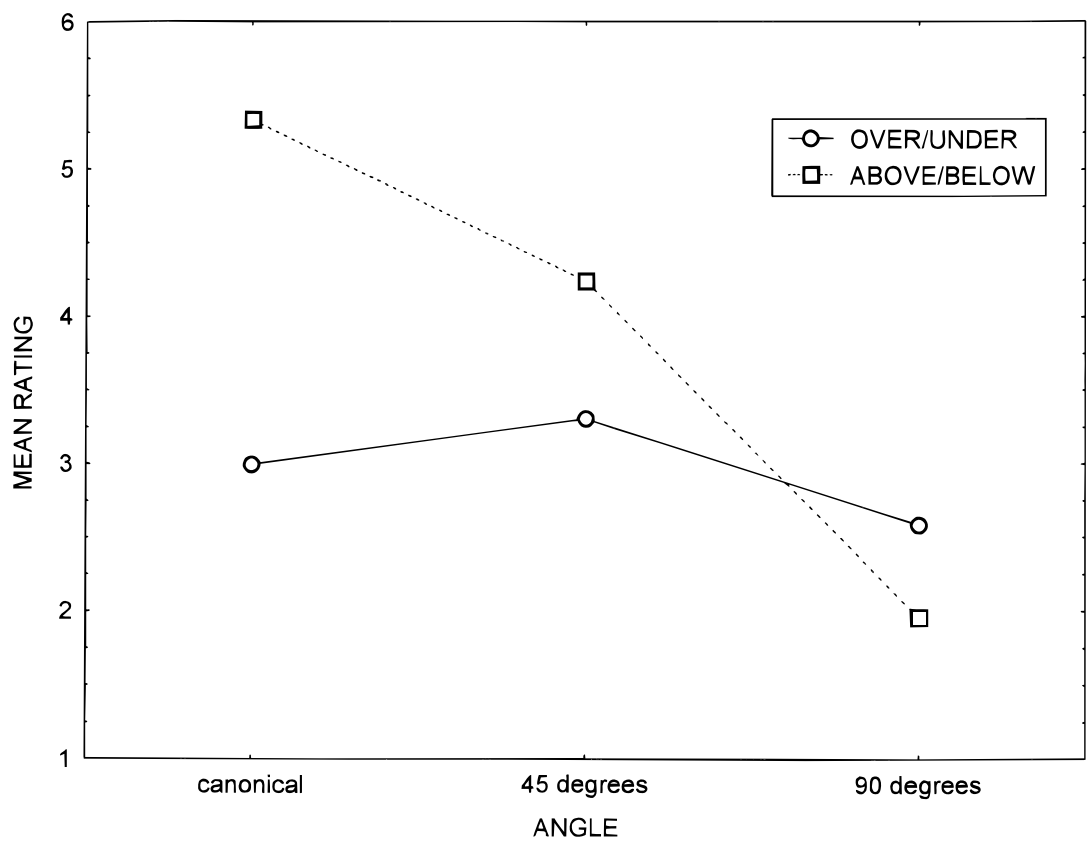

FIG. 4. Interaction between geometry and preposition set for Material Set 1, Experiment 1.

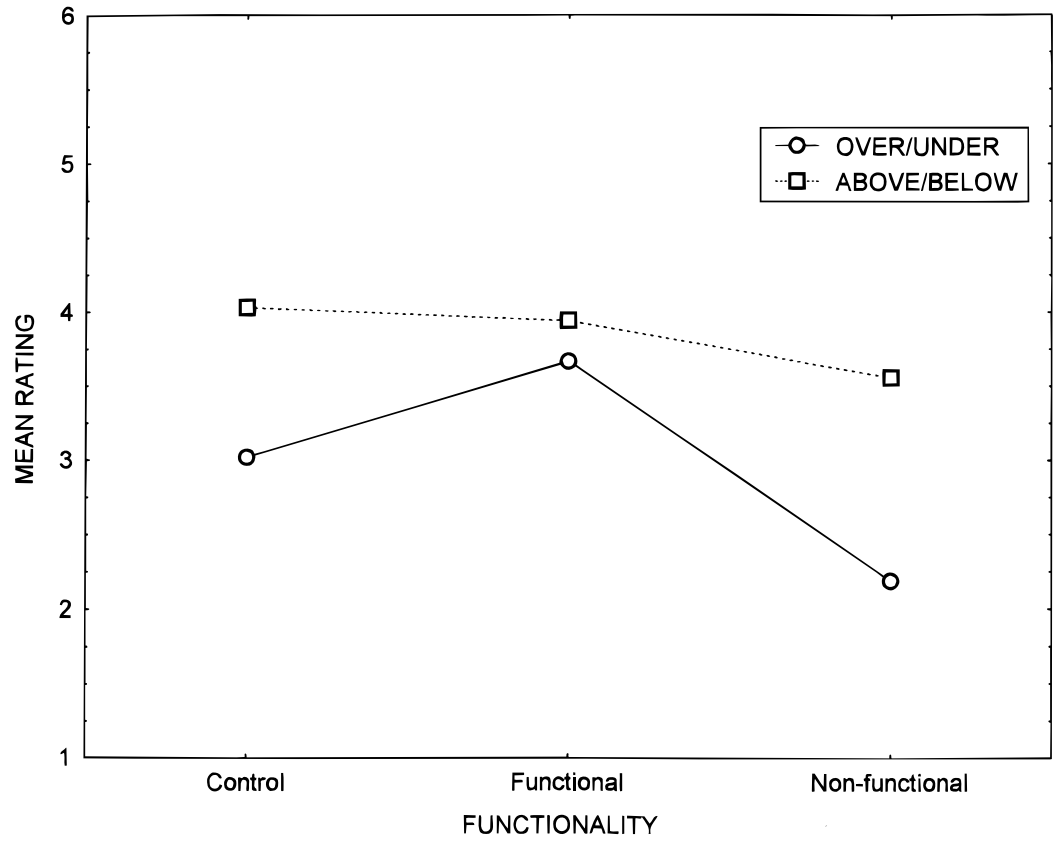

FIG. 5. Interaction between functionality and preposition set for Material Set 1, Experiment 1 . 
above/below were more affected by the angle of the figure than over/under.

Material Set 2. Again a three-way analysis of variance (fully within) was performed on the data using the same variables as those used with Material Set 1. The results of the analysis are displayed in Table 2, and the mean ratings are displayed in the Appendix, Table A2. The results were very similar to those found with the first material set. Main effects of function, angle, and preposition set were found, all in the same direction as those found with Material Set 1 . As before there was also a significant interaction between function and angle.

There were also significant interactions between function and preposition set, and between angle and preposition set. This pattern of results was the same as that found for Material Set 1. Greater function effects were found for over/ under than for above/below while greater geometry effects were found for above/below than for over/under. The three-way interaction between function, angle, and functional/nonfunctional prepositions was also significant. This is displayed in Fig. 6.

\section{Discussion}

The results across material sets 1 and 2 were very similar. Main effects of function and angle were found in both sets, demonstrating that functional relations and geometry are both important factors in determining the appropri- ateness of a preposition to describe a spatial scene.

With regards to geometry, the results were similar to those found in previous studies (Carlson-Radvansky \& Irwin, 1993, 1994; Hayward \& Tarr, 1995; Logan \& Sadler, 1996). When the figure is displaced from the point directly above the central axis of the reference object, appropriateness ratings for suitable spatial prepositions are reduced. However, this study also revealed a main effect of function of magnitude similar to that found for the geometric manipulation. Scenes where the functional relation between objects held were associated with significantly higher appropriateness ratings than scenes where no such relation was present. Furthermore, when an object was shown to be not fulfilling its function, ratings were lower still.

Importantly, the results also indicate that functional relations influence comprehension even when the geometric constraint appears to hold clearly. The effect of functional relations was present with both sets of materials at all three positions, not just in the marginal geometric cases, suggesting that functional relations do not just come into play when the prototypical region does hold (Landau \& Munnich, 1998).

While both geometry and function were shown to be important factors overall in the first experiment, the results also indicate that prepositions are not influenced by geometry and function to the same extent. The ratings of over and

TABLE 2

Results of Three-Way ANOVA for Experiment 1, Material Set 2

\begin{tabular}{lccc}
\hline \multicolumn{1}{c}{ Source } & df and F value & MS $_{e}$ & Significance \\
\hline Functionality (F) & $\mathrm{F}(2,70)=96.23$ & 1.01 & $* * *$ \\
Angle (A) & $\mathrm{F}(2,70)=164.71$ & 1.64 & $* * *$ \\
Preposition set (PS) & $\mathrm{F}(1,35)=119.45$ & 2.18 & $* * *$ \\
$(\mathrm{~F}) \times(\mathrm{A})$ & $\mathrm{F}(4,140)=10.47$ & 1.99 & $* * *$ \\
$(\mathrm{~F}) \times(\mathrm{PS})$ & $\mathrm{F}(2,70)=35.86$ & 0.75 & $* * *$ \\
$(\mathrm{~A}) \times(\mathrm{PS})$ & $\mathrm{F}(2,70)=46.52$ & 0.52 & $* * *$ \\
$(\mathrm{~F}) \times(\mathrm{A}) \times(\mathrm{PS})$ & $\mathrm{F}(4,140)=7.26$ & 0.25 & $* * *$ \\
\hline
\end{tabular}



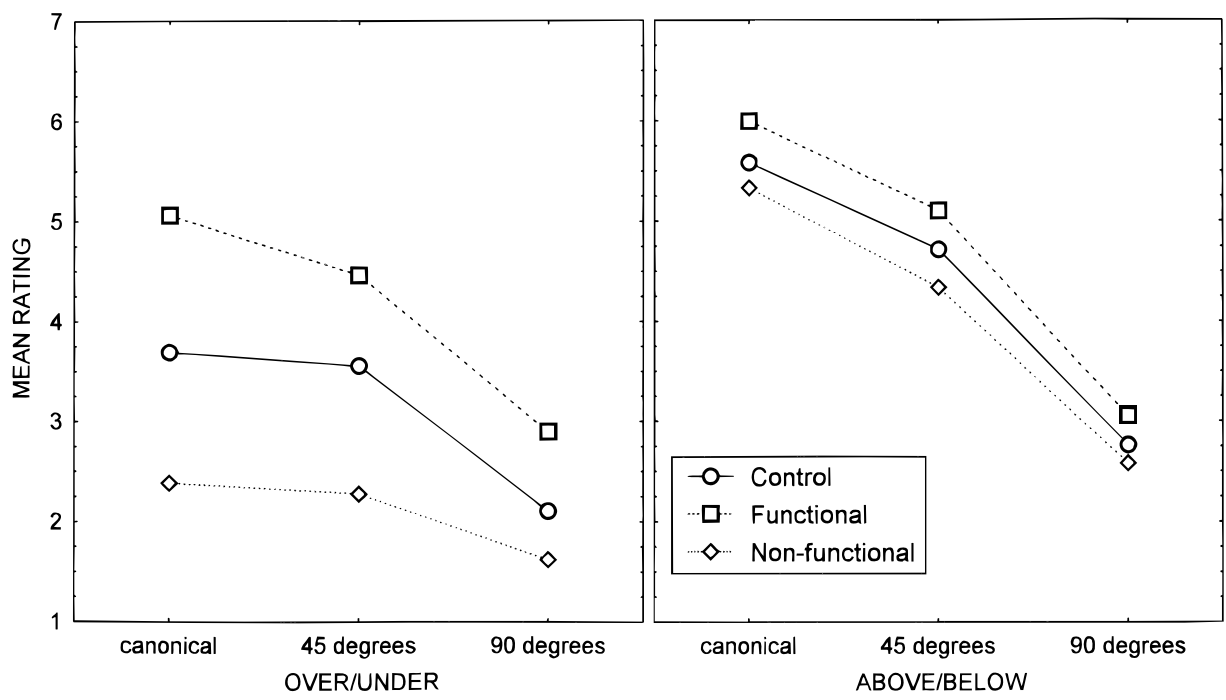

FIG. 6. Interaction among function, geometry, and preposition set for Material Set 2, Experiment 1.

under across both sets of materials were found to be mainly influenced by functionality while above and below were mainly influenced by geometry. This is the first demonstration that geometric and extrageometric relations affect prepositions differentially.

While the results were consistent across both sets of materials, the effects observed with Material Set 1 may only be present with objects which have particular functions. Although the effects were present across a range of types of scenes, in all cases the protecting surfaces were fulfilling the functions they were designed to fulfill. Given that Coventry et al. (1994) report objectknowledge differences for in depending on the appropriateness of the object, the second experiment was designed to see whether the function effects found in Experiment 1 (Material Set 1) are object-specific, or whether they are still present when objects are fulfilling a function in context that is different from their usual function.

\section{EXPERIMENT 2}

The second experiment aimed to establish whether the function effects found in Experiment 1 for the first set of materials are present only for objects which are associated with the function in question, or more generally for any objects fulfilling a recognizable (but noncanoni- cal) function in context. For example, it was of interest to establish whether function effects are still present when a suitcase is used to shelter someone from the rain as compared to when an umbrella is used.

\section{Design}

The experimental design was similar to that used in Experiment 1. In addition to the manipulation of geometry and functionality as before, the appropriateness of the figure as a protecting object was also manipulated. For example, either an umbrella (functionally appropriate object) or a suitcase (functionally inappropriate object) was used as the source of protection from rain. The predictions were the same as those for Experiment 1 . Additionally, it was predicted that, if function effects are object-specific, then function effects should be present only with the functionally appropriate objects. Alternatively, if functional relations are nonspecific, and determined by situation-specific context, then function effects should be present for both appropriate and inappropriate objects.

\section{Method}

Participants. Forty A-level students from a local sixth-form college (age range 17-18) were paid for their participation in the experiment. 
All participants were native speakers of English. Data from one participant had to be eliminated because he did not finish the experiment.

Materials. The materials for the experiment consisted of a total of 288 pictures and were based on the first set of (four) pictures used in Experiment 1. This time each picture had 18 variants (three levels of geometry, three levels of functionality, and two levels of appropriateness), making a total of 144 pictures. Each picture was printed twice, once with a pair of sentences (e.g., The man is under/below the umbrella) and once with another pair of sentences (e.g., The umbrella is over/above the man) to test all four prepositions. The inappropriate objects used were a suitcase, book, bucket, and stool substituted for umbrella, visor, hard hat, and shield, respectively. In all cases the dimensions of the inappropriate objects were matched exactly to those of the appropriate objects. See Fig. 7 for some examples of pictures with inappropriate objects.

The test materials were mixed in with materials from other experiments. This was done so that other items would act as filler items, thus adding variety to the materials used and prevent- ing participants from falling into a pattern. As these filler items are not relevant here, they will not be discussed any further.

\section{Procedure}

Participants were run in one large group, and the procedure was essentially the same as that used in Experiment 1 with the following exception. In order to cut down the number of scenes each participant was exposed to, half the participants were given the umbrella/suitcase visor/ book materials (set A) for over and above and the shield/stool hard hat/bucket (set B) for under and below. The other half was given the reverse.

The materials were divided into two parts (although no break was given). One copy of each picture (with one pair of prepositions) appeared in part one, and the other copy of the picture (with the other pair of prepositions) appeared in part two. Half of the participants saw part one first, and the other half saw the second part first (though participants were not aware of the two parts). Additionally, scenes with the inappropriate and appropriate objects always occurred in different parts. The materials were
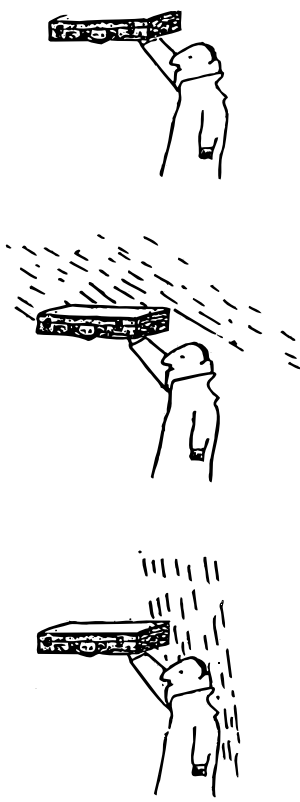
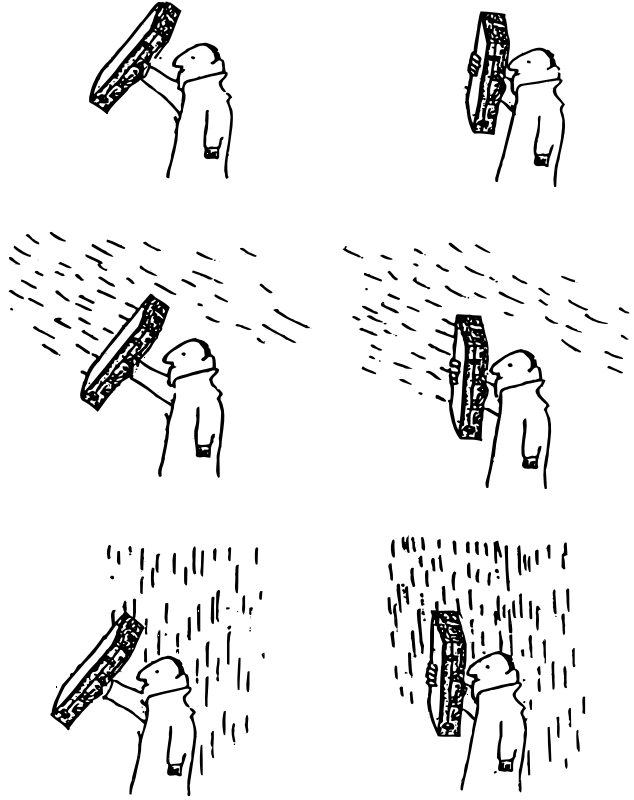

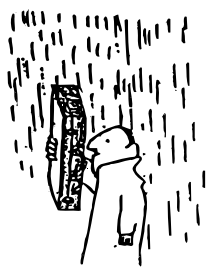

FIG. 7. Examples of materials used in Experiment 2. 
also randomized, ensuring that consecutive pictures were never identical. Each participant had a different grouping and randomization. The end result of this process meant that the possibility of priming effects was minimized.

\section{Results and Discussion}

A four-way analysis of variance (fully within) was performed on the rating data. The mean ratings are displayed in the Appendix, Table A3, and the results of the analysis are displayed in Table 3. The variables were appropriateness of figure (appropriate versus inappropriate figure), function (e.g., functional, nonfunctional, and control), angle (canonical, $45^{\circ}$, or $90^{\circ}$ ), and preposition set (over/under versus above/below).

The results of analysis mirror those found in Experiment 1. Main effects of function, geometry, and preposition set were found in the same directions as before. The pattern of interactions between these variables also mirrored those reported for Experiment 1. As Figs. 8 and 9 illustrate, again over/under were found to be more influenced by functional relations than above/ below, and conversely, above/below are more influenced by geometry than over/under.
Of most interest in the analyses was the significant main effect of appropriateness of figure. Scenes involving functionally appropriate objects were rated significantly higher than those involving functionally inappropriate objects. This pattern of results is in line with those found for in by Coventry et al. (1994). However, in the present experiment this variable did not interact with any of the main variables of interest, so the effect of appropriateness in isolation is open to a number of interpretations. Importantly, function and geometry effects were found for both the appropriate and the inappropriate objects, indicating that function effects are not limited to objects which are shown to be fulfilling their object-specific function but also occur when objects are being used in a way that deviates from their usual function. This will be discussed later in the general discussion.

\section{EXPERIMENT 3}

The first two experiments have established that, when a single frame of reference is involved (or where frames of reference coincide), functional relations are important determinants of the rating of the prepositions. There is also a

TABLE 3

Results of Four-Way ANOVA for Experiment 2

\begin{tabular}{|c|c|c|c|}
\hline Source & df and $F$ value & $\mathrm{MS}_{e}$ & Significance \\
\hline Appropriateness of figure (AF) & $\mathrm{F}(1,34)=15.22$ & 0.85 & $* *$ \\
\hline Functionality (F) & $\mathrm{F}(2,70)=22.34$ & 3.22 & $* * *$ \\
\hline Angle (A) & $\mathrm{F}(2,70)=74.40$ & 3.56 & $* * *$ \\
\hline Preposition set (PS) & $\mathrm{F}(1,35)=39.37$ & 6.83 & $* * *$ \\
\hline$(\mathrm{AF}) \times(\mathrm{F})$ & $\mathrm{F}(2,70)=0.79$ & 0.71 & ns \\
\hline$(A F) \times(A)$ & $\mathrm{F}(2,70)=1.42$ & 0.49 & ns \\
\hline$(\mathrm{F}) \times(\mathrm{A})$ & $\mathrm{F}(4,140)=4.66$ & 0.55 & $*$ \\
\hline$(\mathrm{AF}) \times(\mathrm{PS})$ & $\mathrm{F}(1,35)=2.25$ & 1.57 & ns \\
\hline$(\mathrm{F}) \times(\mathrm{PS})$ & $\mathrm{F}(2,70)=8.52$ & 0.91 & $* *$ \\
\hline$(\mathrm{A}) \times(\mathrm{PS})$ & $\mathrm{F}(2,70)=42.20$ & 2.24 & $* * *$ \\
\hline$(\mathrm{AF}) \times(\mathrm{F}) \times(\mathrm{A})$ & $\mathrm{F}(4,140)=1.86$ & 0.40 & ns \\
\hline$(\mathrm{AF}) \times(\mathrm{F}) \times(\mathrm{PS})$ & $\mathrm{F}(2,70)=0.63$ & 0.57 & ns \\
\hline$(\mathrm{AF}) \times(\mathrm{A}) \times(\mathrm{PS})$ & $\mathrm{F}(2,70)=0.18$ & 0.42 & ns \\
\hline$(\mathrm{F}) \times(\mathrm{A}) \times(\mathrm{PS})$ & $\mathrm{F}(4,140)=0.85$ & 0.36 & ns \\
\hline$(\mathrm{AF}) \times(\mathrm{F}) \times(\mathrm{A}) \times(\mathrm{PS})$ & $\mathrm{F}(4,140)=0.86$ & 0.35 & ns \\
\hline
\end{tabular}




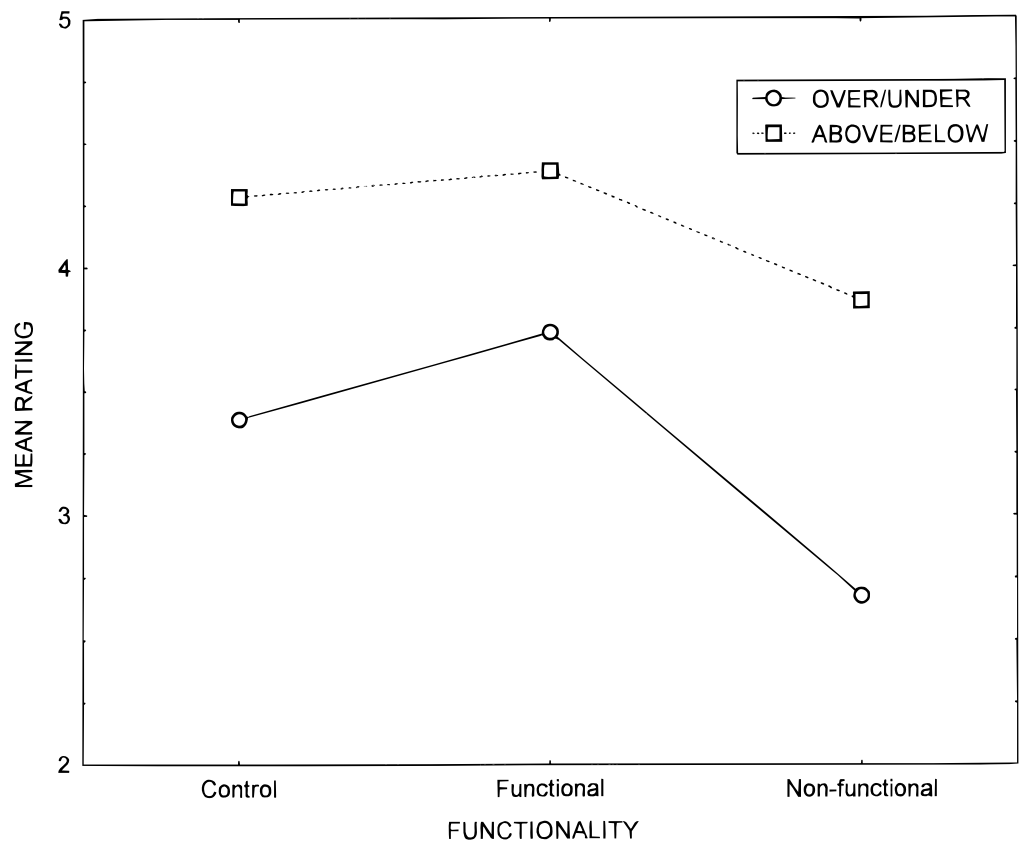

FIG. 8. Interaction between functionality and preposition set in Experiment 2.

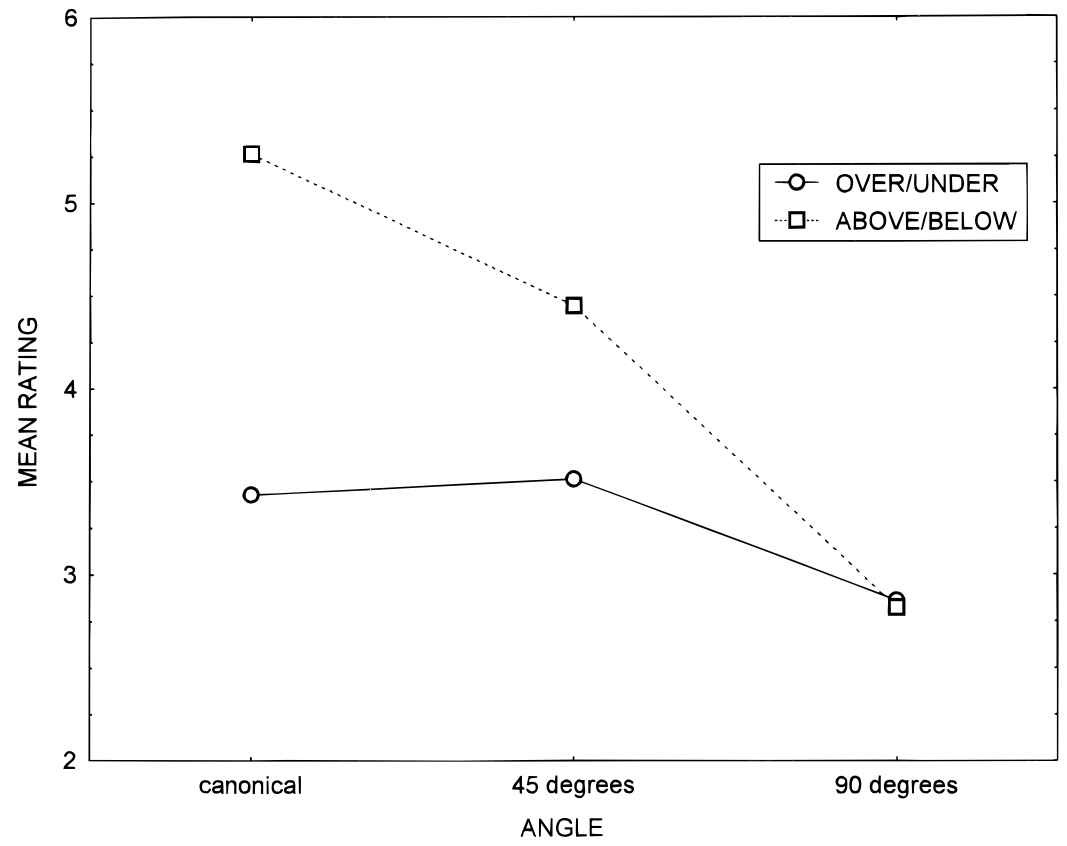

FIG. 9. Interaction between geometry and preposition set in Experiment 2 . 
consistent difference between over/under and above/below. Over/under are more influenced by functional relations than above/below, and conversely it would appear that above/below are more influenced by geometry. The purpose of this experiment was to examine the relative effects of these variables by manipulating geometry in such a way as to provide coincidence or conflict between frames of reference. For example, the scenes in Fig. 10 illustrate a Viking holding a shield, but unlike the geometric manipulation of the position of the figure in Experiments 1 and 2, the rotation of the ground leads to conflicts of reference frames. With scenes on the left, the extrinsic (gravitational) and intrinsic (object-centered) frames of reference coincide. With scenes in middle and on the right, the extrinsic and intrinsic frames do not coincide but conflict. For scenes on the left, one can say that the shield is above the Viking for both frames of reference, but for scenes in the middle and on the right, the shield is above the Viking is appro-
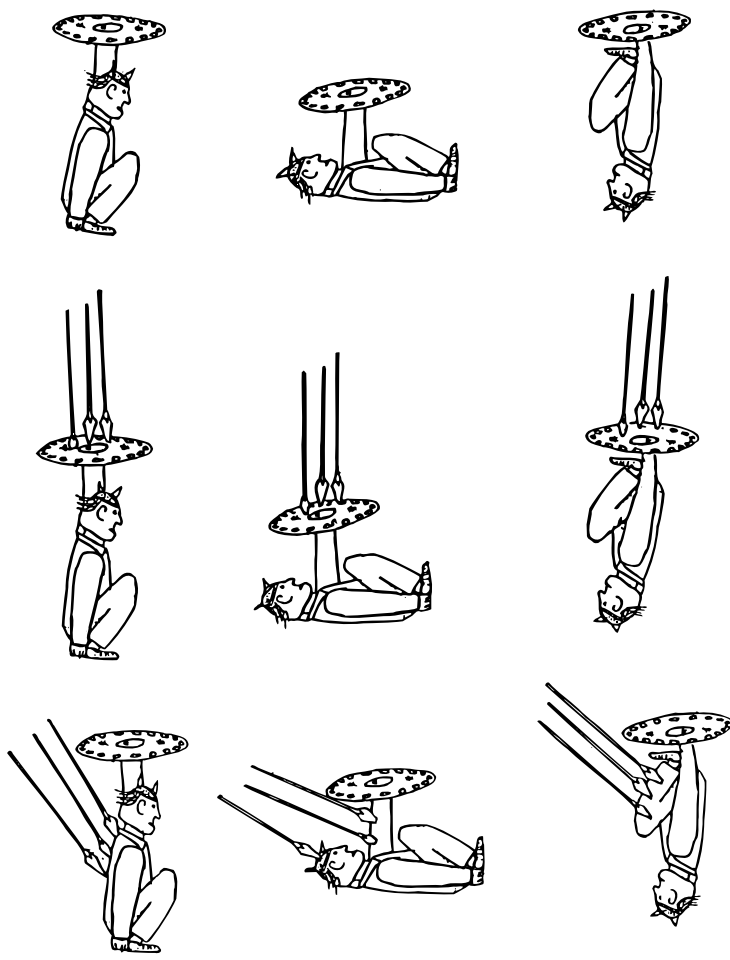

FIG. 10. Examples of materials used in Experiment 3. priate for the extrinsic frame of reference but inappropriate for the intrinsic frame of reference.

Given the findings that geometry has a much bigger effect on the rating of above/below than over/under (and vice versa for functionality), we predicted that conflict between reference frames (a geometric manipulation) should similarly influence the ratings of above/below more than over/under. In the literature on frames of reference, above and over have been classified together as projective prepositions (prepositions which also convey information about the direction in which one object is located with respect to the other), and therefore it has been tacitly assumed that they are influenced by frame of reference effects in the same way. This experiment will determine whether this is the case.

\section{Design}

The experiment was designed to test for the differential effects of function and frame of reference on participants' ratings of the appropriateness of sentences to describe a series of pictures. The variables manipulated included three levels of geometry of the ground and three levels of functionality. The figure was always placed in the same location and with the same angle (cf. Carlson-Radvansky \& Irwin, 1994). One level of geometry of the reference object (ground) yielded a unique frame of reference, i.e., the intrinsic, absolute, and relative frames of reference were the same. The other two levels of geometry of the reference object yielded a conflicting frame of reference between intrinsic frame of reference and absolute frame of reference. The extrinsic frame of reference was always collapsed with the relative frame of reference.

The predictions were that if conflicting frame of reference had an effect on the comprehension of these prepositions, the participants would give the highest ratings to a sentence when it described a picture where there was no conflicting frame of reference (i.e., the intrinsic, the absolute, and the extrinsic reference frames were the same). We were also predicting that if functional relations had an effect, participants' ratings for a particular sentence would be higher when it described a picture depicting a functional relation between objects than when the 
functional relation was not present. However, following previous results, we also predicted that over and under would be more sensitive to functional relations than above and below, and that above and below would be more affected by conflict of frame of reference than over and under.

\section{Method}

Participants. Thirty-six undergraduate students from the University of Plymouth participated in this experiment as an extra credit option in a Psychology course. All participants were native speakers of English. Data from one participant had to be eliminated because he did not finish the experiment.

Materials. The materials for the experiment consisted of a total of 72 pictures and were based on four types of pictures. Each type of picture had nine variants (three levels of rotation of the referent object and three levels of functionality). Each picture was printed twice, once with a pair of sentences (e.g., The shield is over/ above the Viking) and once with another pair of sentences (e.g., The Viking is under/below the shield) to test all four prepositions. All materials involved the use of appropriate figures.

All pictures depicted a man using an object to protect himself from a falling object/objects (as in Experiments 1 and 2). For each picture there were three levels of functionality and geometry (as in Experiments 1 and 2). However, for the geometry manipulation this time the man in the picture was positioned upright, lying on the floor, or upside-down. Half of the pictures had the man rotated to the left, and in the other half the man was rotated to the right. See Fig. 10 for an example of all nine levels of picture.

Again the experimental items were mixed in with 88 filler items from other experiments in order to give variety to the experiment and prevent participants from falling into a pattern.

\section{Procedure}

The procedure for testing was the same as that used for Experiments 1 and 2. This time the experimental sessions lasted around $25 \mathrm{~min}$.

The test materials were divided into two parts. One copy of each picture (with one pair of prepositions) appeared in part one, and the other copy of the picture (with the other pair of prepositions) appeared in part two. Half of the participants saw part one first, and the other half saw the second part first (though participants were not aware of the two parts). In each part, half of the materials contained the prepositions over/ above, and the other half contained the prepositions under/below. The materials for each part together with the fillers were completely randomized with the only restriction being that there were never two picture materials from the same experiment paired consecutively. This made sure that participants never rated the same prepositions (over/above or under/below) consecutively. Each participant received the materials randomized in a different order.

\section{Results and Discussion}

A three-way analysis of variance (fully within) was performed on the rating data. The mean ratings (collapsed across materials) are displayed in the Appendix, Table A4, and results of the analysis are displayed in Table 4. The variables were function (e.g., functional, nonfunctional, and control), angle (canonical, $90^{\circ}$, or $180^{\circ}$ ), and preposition set (over/under versus above/below).

A main effect of function was found. Followup analysis using Tukey HSD tests revealed that the nonfunctional scenes (mean rating $=5.61$ ) were rated significantly lower than both the functional (mean $=6.11$ ) and control scenes (mean $=6.06)$. There was also a main effect of angle. The canonical scenes $($ mean $=6.08)$ were rated significantly higher than either the $90^{\circ}$ $($ mean $=5.79)$ or $180^{\circ}$ scenes $($ mean $=5.91)$. A main effect was found for preposition set in the same direction found in the previous experiment.

No significant interaction was observed between function and angle, but interactions were found between the preposition set and angle and preposition set and function. These interactions are displayed in Figs. 11 and 12.

The interaction between geometry and preposition set illustrates a conflict of frame of reference effect is present for above/below; the greater the conflict (rotation of ground), the lower the ratings for these prepositions. The scenes where the ground is rotated $90^{\circ}$ and $180^{\circ}$ involve a 


\section{TABLE 4}

Results of Three-Way ANOVA for Experiment 3

\begin{tabular}{lccc}
\hline \multicolumn{1}{c}{ Source } & df and F value & $\mathrm{MS}_{e}$ & Significance \\
\hline Functionality (F) & $\mathrm{F}(2,68)=19.29$ & 0.81 & $* * *$ \\
Angle (A) & $\mathrm{F}(2,68)=7.85$ & 0.56 & $* *$ \\
Preposition set (PS) & $\mathrm{F}(1,34)=34.45$ & 2.50 & $* * *$ \\
$(\mathrm{~F}) \times(\mathrm{A})$ & $\mathrm{F}(4,136)=1.20$ & 0.13 & $\mathrm{~ns}$ \\
$(\mathrm{~F}) \times(\mathrm{PS})$ & $\mathrm{F}(2,68)=17.41$ & 0.81 & $* * *$ \\
$(\mathrm{~A}) \times(\mathrm{PS})$ & $\mathrm{F}(2,68)=11.39$ & 0.29 & $\mathrm{~ns}$ \\
$(\mathrm{~F}) \times(\mathrm{A}) \times(\mathrm{PS})$ & $\mathrm{F}(4,136)=1.30$ & 0.13 & \\
\hline
\end{tabular}

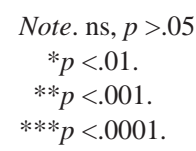

conflict between the object-centered and environment-centered frames of reference, and therefore it is unsurprising that the ratings for these scenes are lower than those for the canonical scenes where there is a correspondence between frames of reference. However, for over/under a significant drop in rating for the $90^{\circ}$ was in evidence compared to the other two positions. No significant difference was present between the canonical and $180^{\circ}$ scenes. This result makes sense if one interprets it from a functional perspective. When the ground is in the supine position, the surface area of the figure is not large enough to ensure that the rain, for example, will not drip off the umbrella and wet the man. Therefore the figure is not able to fulfil its function due to its inappropriate size, and as a consequence the functional scenes and control

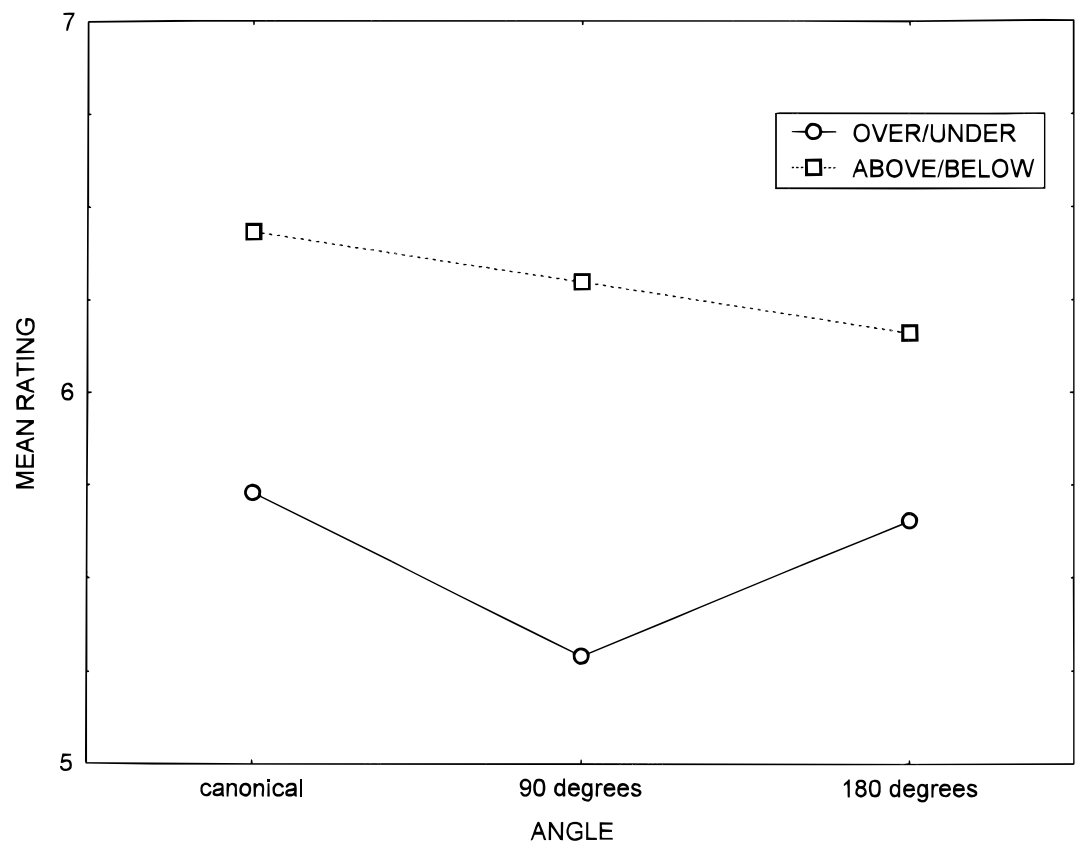

FIG. 11. Interaction between geometry and preposition set in Experiment 3. 


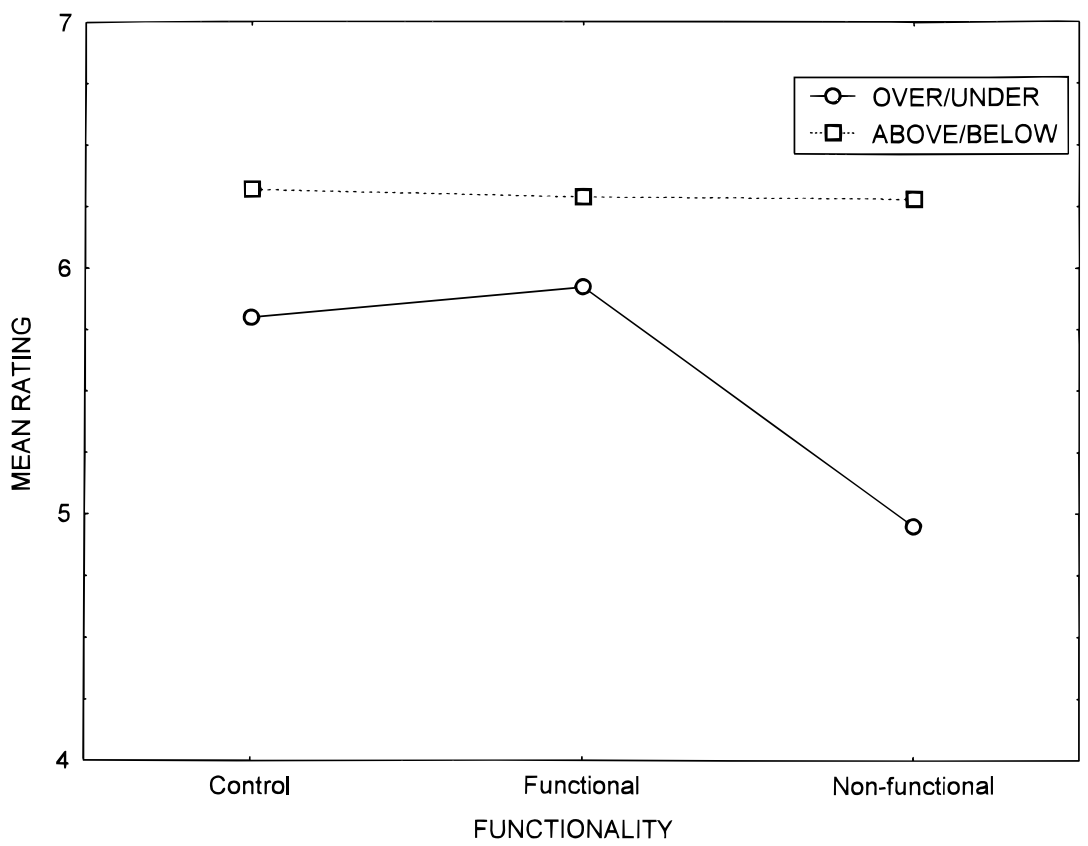

FIG. 12. Interaction between functionality and preposition set in Experiment 3.

scenes are much nearer the ratings for the nonfunctional scenes where the figure is not fulfilling its function, although the nonfunctional ratings were still significantly lower than the other two levels of functionality. If this explanation is correct one might expect that the use of larger objects would cause this effect to disappear.

In line with Experiments 1 and 2, this experiment produced differences in the influence of function and geometry on over/under versus above/below, although in magnified form. The significant interaction between functionality and preposition set revealed that over/under were influenced greatly by functionality while no function effects were found for above/below at all.

\section{GENERAL DISCUSSION}

The pattern of results across the experiments demonstrates the importance of both geometric and functional relations as factors influencing the appropriateness ratings of spatial prepositions. Main effects of function and geometry were found in all three experiments. Overall, the degree of alignment of figure and ground with reference to the gravitational axis influences the ratings of superior and inferior relations, as has been found previously (Carlson-Radvansky \& Irwin, 1993, 1994; Hayward \& Tarr, 1995; Logan \& Sadler, 1996). In addition, functional relations in the present studies also influence the ratings of superior and inferior relations. When a figure is shown to be fulfilling its function in context ratings increase over a control, while flouting of function leads to a decrease in ratings. These results demonstrate that functional relations are important in the comprehension of spatial prepositions. The results also go some way toward specifying what specific types of object-knowledge effects previously subsumed under the banner of functional relations actually influence ratings. In particular the results show the effect of functionality in relation to two different types of object knowledge- the functions that objects usually have (for both protecting and containment functions) and how objects are functioning (noncanonically) in context.

Given that geometry and function are both important determinants of the ratings given in the present studies, the results for the first time shed some light on the interplay between geometric 
and extrageometric variables. Landau and Munnich's (1998) suggestion that extrageometric factors come into play only in cases where the geometric constraint does not clearly hold would not appear to be supported by the present data. In all three experiments the influence of functional relations was in evidence even when the prototypical geometric relation was depicted, indicating that extrageometric factors may play more than the secondary role proposed by Landau and Munnich.

The results also provide evidence for the first time that prepositions are differentially influenced by function and geometry. While most previous studies have made the assumption that over and above, and under and below have similar meanings (e.g., Bennett, 1975; Logan \& Sadler, 1996), the pattern of results here illustrates important differences between the two preposition sets. In all three experiments, interactions between geometry, function, and preposition set were found. Over and under were found to be more influenced by the functional manipulation than above and below, and conversely above and below were more influenced by geometric manipulations than over and under. Most striking were the differences between preposition sets when conflict of reference frames was involved in Experiment 3; no effects of conflict of reference frame were found for over/under, and no effects of function were found for above/below.

\section{Over, under, above, and below revisited}

Armed with the present set of results, we can begin to develop an account of the meaning of over, under, above, and below which involves the integration of both geometric and extrageometric variables. A useful place to start is with the reexamination of how geometric approaches in isolation break down in the explanation of the present data. As Logan and Sadler (1996) offer an unusually well-specified account of how geometric regions operate which is compatible with less well-specified linguistic accounts (e.g., Bennett, 1975; Herskovits, 1986), we can begin with a consideration of the limitations of their approach. According to Logan and Sadler, spatial templates are used to map onto visual scenes in order to apprehend both spatial relations and spatial expressions. A spatial template is a spatially defined representation in the form of a grid that specifies areas of relative acceptability of a figure positioned relative to a ground (see also Hayward \& Tarr, 1995). When the spatial template is centered on the reference object and aligned with its reference frame, it specifies the goodness with which located objects in different positions exemplify the associated relation. Furthermore, it is assumed that there are different spatial templates for different spatial terms, and when polysemy exists, there is a different spatial template for each polyseme. Additionally, when there are a number of reference frames which are possible for a visual scene, Logan and Sadler (see also Carlson-Radvansky \& Logan, 1997) claim that these are activated simultaneously and in parallel during comprehension.

Within this framework, the most appropriate regions on the template for over and above are directly above the ground, while the highest ratings for under and below are directly below the ground (as is the case in linguistic accounts such as Bennett, 1975). As already indicated, if we were to follow the line taken by Landau and Munnich (1998), then functional relations should only have an influence on ratings when the position of the figure on the template lies in a region with a low acceptability rating. In that case, one could argue that extrageometric information comes into play when the geometric constraints do not clearly hold. However, we have seen that the functionality of an object influences the ratings of prepositions in cases where the optimal place on the spatial template maps onto the scene (e.g., see Figs. 3 and 6). This suggests that multiple constraints are used to evaluate the appropriateness with which spatial expressions map onto visual scenes.

While a geometric analysis of the type proposed by Logan and Sadler (1996; see also Regier, 1996) offers a means of capturing geometric relations in a precise way, this is only one type of constraint which relates to use and comprehension. Other constraints come in the form of the functions that objects have (which may well be lexicalized), how objects are functioning in context, and the wider context in which the language and/or visual scene occurs. Although 
some of these constraints are driven by language, ultimately the situation-specific meaning of spatial expressions also relies on nonlinguistic information present in the visual context and conceptual information which is best characterized nonlinguistically. These sources of information may be integrated on-line in a mental model of the type proposed by Garrod and Sanford (1989) and Coventry (1998), the output of which leads to a situation-specific use/comprehension of a spatial expression. The view here is in line with a growing literature which demonstrates that multiple constraints, both linguistic and nonlinguistic, influence comprehension on line (e.g., Sanford and Garrod, 1998; Sedivy, Tanenhaus, Chambers, \& Carlson, 1999).

In order to see how this type of model operates, we can reconsider the data set. Experiment 1 has shown that information regarding the protecting function of a figure influences ratings of spatial prepositions. One possible account of this finding involves an extension of the lexical entries for figure and ground present in the sentence to be rated. Given that an umbrella, for example, has a protecting function, it could be argued that the presence of rain in the visual scene becomes relevant driven by the lexicalized function for the noun. However, while the lexical entries for the nouns occurring in the expression are undoubtedly important, the lexical entries combined do not provide an adequate account of the data in the present experiments, even with this added (pragmatic) extension. The same pattern of results observed in Experiment 1 was found in Experiment 2 when figures were used which do not have the lexicalized function depicted in the visual scene. The relevance of rain in this case is unlikely to be driven by the lexical entry for suitcase (instead of an umbrella) but rather is present in the visual scene being described. Therefore, co-occurrence relations/distributional relations alone of the type advocated more generally by Burgess and Lund (1997) and Landauer and Dumais (1997) are unlikely to provide an adequate account of meaning of spatial expressions without consideration of nonlinguistic variables.

Although one can outline a number of different variables which need to be invoked to ac- count for situation-specific meaning of spatial expressions, the results of all three experiments indicate that different factors have different weighted influences on over/under versus abovel below. In Experiments 1 and 2, although both preposition sets were influenced by the geometric and functional manipulations, over/under were more heavily influenced by functionality while above/below were more heavily influenced by geometry. Most striking was the absence of a conflict of frame of reference effect for over/under in Experiment 3; over/under was affected by functionality alone while above/ below exhibited clear frame of reference conflict effects but no functionality effects. These results are compatible with the view that objectknowledge is more important for over/under than for above/below. While the results for above/below support Logan and Sadler's (1996) claim that multiple spatial templates may be imposed on the visual scene centered on the position of the ground, the results for over and under suggest that there is no need to use multiple spatial templates for these terms as early processing of information in the scene (e.g., the rain) clearly primes the construction or selection of the absolute (environment-centered) frame. Therefore Logan and Sadler's (1996) claim that spatial templates are computed in parallel may not hold for over/under. Thus we can begin to see that different sources of information may be used for different preposition sets.

The explanation for the pattern of differences for over/under versus above/below in terms of weightings of different linguistic and nonlinguistic constraints does not preclude the influence of functional factors for above/below or geometric factors for over/under. Indeed Logan and Sadler (1996) found that over and above, and under and below, behaved in the same way across a series of experiments involving abstract objects without any salient functional properties in the absence of a context. If objects are present without any functions, such as the abstract objects used in the experiments of Logan and Sadler, it is unsurprising that over/under and above/below appear to behave in the same way. Where there are no object properties of relevance in a scene, geometric properties alone can 
still be used to evaluate the appropriateness of these spatial terms. On the other hand, CarlsonRadvansky et al. (1999) have shown that functional relations can influence the comprehension of above in cases where this is the salient manipulation, and functional influences on above/ below were present in the first two experiments here, although the effects were smaller than for over/under.

The difference in the relative importance of geometric and extrageometric factors for different spatial terms is likely to relate to the extent to which prepositions may be regarded as polysemous. While above/below are often treated as having one sense each (e.g., locative superior/ locative inferior; Bennett, 1975), over and under by contrast are highly polysemic, as has been shown by Brugman (1988) and Lakoff (1987). Indeed, three central senses of over have been proposed by Brugman (1988) — the "above" schema (where the figure is higher than but not in contact with the ground), the "cover" schema (where the figure covers the ground and is usually above and in contact with the ground as in the tablecloth is over the table), and the "aboveacross" schema (where the figure is moving on a path above and extending beyond the boundaries of the ground as in the plane flies over the bridge). Although these are the central senses proposed by Brugman, she outlines dozens of other possible senses which are all lexicalized. If we follow Logan and Sadler and assume that there exists a spatial template for each polyseme, it is not surprising that over is more subject to extrageometric influences than above given that object knowledge needs to be accessed early on to work out which sense or template is appropriate in context.

As an alternative to the multiple representation of spatial templates or senses for over in the lexicon, it could be that the large amount of polysemy proposed for over by Brugman (1988) and Lakoff (1987) may be the result of the sensitivity of this term to contextual modulation. Rather than using extrageometric information early on in processing to select the appropriate sense/spatial template, object knowledge used early on may allow the generation (sense creation) of a situation-specific meaning without the need to separately represent a massive number of senses in the lexicon. Many of the multiple senses in the analysis given by Brugman and Lakoff naturally fall out of this approach without the need to lexicalize. For example, Brugman (1988) assumes that the differences in the presence of contact in the tablecloth is over the table versus the hand is over the table is a result of having two different lexicalized senses for over. The alternative view is that a tablecloth has a lexicalized covering function, and therefore it is expected that the tablecloth will be in contact with the table. Therefore it is not the preposition which is explicitly marked or unmarked for contact, but rather the situationspecific context primes a model where contact may be clearly required, disallowed, or either allowed or disallowed. In this way the variety in the situation-specific meanings of over is a result of sensitivity to contextual modulation and constraints provided by the context.

We have begun to explore an account of the differences between spatial prepositions in the extent to which they are influenced by geometric and extrageometric constraints which must reside in both linguistic and nonlinguistic domains. While above and below would appear to be more purely geometrically determined, and are perhaps more completely specified in terms of spatial templates, over and under by contrast would appear to be less well specified in the lexicon and more subject to contextual modulation. However, the account we have put forward needs to be further fleshed out. For example, the issue of sense selection versus sense creation for spatial terms remains unresolved in terms of the present data set. In particular, it is of interest to examine how processing takes place on line in real time. Nevertheless, the results for the first time indicate that multiple constraints are needed, and further that a hitherto unrecognized spatial preposition classification may be present in English. This classification is a continuum from prepositions like above and below, which are primarily geometrically determined, to those like in, on, over, and under, which are influenced by extrageometric relations and in particular factors which have to do with what objects are for and what they are doing, as well as where they are. 


\section{APPENDIX}

TABLE A1

Mean Ratings for Material Set 1, Experiment 1

\begin{tabular}{|c|c|c|c|c|c|c|}
\hline & \multicolumn{2}{|c|}{ Canonical } & \multicolumn{2}{|c|}{45 degrees } & \multicolumn{2}{|c|}{90 degrees } \\
\hline & Over/under & Above/below & Over/under & Above/below & Over/under & Above/below \\
\hline Control & $2.97 / 3.20$ & $5.89 / 5.35$ & $3.34 / 3.58$ & $4.57 / 4.41$ & $2.72 / 2.31$ & $1.95 / 2.03$ \\
\hline Functional & $3.57 / 3.57$ & $5.58 / 5.18$ & $4.03 / 4.10$ & $4.43 / 4.36$ & $3.49 / 3.26$ & $2.04 / 2.07$ \\
\hline Nonfunctional & $2.14 / 2.51$ & $5.12 / 4.89$ & $2.37 / 2.41$ & $3.74 / 3.93$ & $1.99 / 1.73$ & $1.70 / 1.96$ \\
\hline
\end{tabular}

TABLE A2

Mean Ratings for Material Set 2, Experiment 1

\begin{tabular}{|c|c|c|c|c|c|c|}
\hline & \multicolumn{2}{|c|}{ Canonical } & \multicolumn{2}{|c|}{45 degrees } & \multicolumn{2}{|c|}{90 degrees } \\
\hline & Over/under & Above/below & Over/under & Above/below & Over/under & Above/below \\
\hline Control & $3.52 / 3.85$ & $5.62 / 5.54$ & $3.44 / 3.66$ & $4.86 / 4.58$ & $2.20 / 2.01$ & $3.01 / 2.51$ \\
\hline Functional & $5.02 / 5.10$ & $6.03 / 5.96$ & $4.43 / 4.49$ & $5.29 / 4.92$ & $3.13 / 2.66$ & $3.24 / 2.87$ \\
\hline Nonfunctional & $2.16 / 2.60$ & $5.56 / 5.10$ & $2.29 / 2.26$ & $4.40 / 4.28$ & $1.62 / 1.63$ & $2.68 / 2.48$ \\
\hline
\end{tabular}

TABLE A3

Mean Ratings for Experiment 2

\begin{tabular}{|c|c|c|c|c|c|c|}
\hline & \multicolumn{2}{|c|}{ Canonical } & \multicolumn{2}{|c|}{45 degrees } & \multicolumn{2}{|c|}{90 degrees } \\
\hline & Over/under & Above/below & Over/under & Above/below & Over/under & Above/below \\
\hline \multirow[t]{2}{*}{ Control } & $3.58 / 3.87$ & $5.49 / 5.21$ & $3.98 / 3.99$ & $4.73 / 4.63$ & $3.37 / 2.74$ & $2.92 / 2.92$ \\
\hline & $3.26 / 3.38$ & $5.52 / 5.27$ & $3.60 / 3.54$ & $4.73 / 4.49$ & $2.91 / 2.27$ & $2.65 / 2.65$ \\
\hline \multirow{2}{*}{ Functional } & $3.74 / 4.05$ & $5.51 / 5.47$ & $3.96 / 4.56$ & $4.70 / 4.78$ & $3.88 / 3.11$ & $3.06 / 3.00$ \\
\hline & $3.84 / 3.53$ & $5.63 / 5.10$ & $3.88 / 3.51$ & $4.66 / 4.30$ & $3.36 / 3.28$ & $3.25 / 3.03$ \\
\hline \multirow[t]{2}{*}{ Nonfunctional } & $2.75 / 3.06$ & $4.88 / 4.93$ & $2.85 / 2.80$ & $4.06 / 4.15$ & $2.78 / 2.23$ & $2.84 / 2.42$ \\
\hline & $3.02 / 2.89$ & $5.03 / 4.80$ & $2.59 / 2.74$ & $4.07 / 3.83$ & $2.12 / 2.14$ & $2.45 / 2.67$ \\
\hline
\end{tabular}

Note. Regular characters are for appropriate protecting objects, and italicized characters are ratings for inappropriate protecting objects.

\section{TABLE A4}

Mean Ratings for Experiment 3

\begin{tabular}{|c|c|c|c|c|c|c|}
\hline & \multicolumn{2}{|c|}{ Canonical } & \multicolumn{2}{|c|}{90 degrees } & \multicolumn{2}{|c|}{180 degrees } \\
\hline & Over/under & Above/below & Over/under & Above/below & Over/under & Above/below \\
\hline Control & $5.88 / 6.08$ & $6.50 / 6.39$ & $5.42 / 5.74$ & $6.37 / 6.28$ & $5.63 / 6.04$ & $6.20 / 6.19$ \\
\hline Functional & $6.14 / 6.21$ & $6.47 / 6.42$ & $5.49 / 5.73$ & $6.29 / 6.20$ & $5.99 / 5.99$ & $6.16 / 6.20$ \\
\hline Nonfunctional & $4.91 / 5.15$ & $6.61 / 6.21$ & $4.47 / 4.89$ & $6.41 / 6.24$ & $4.88 / 5.38$ & $6.12 / 6.09$ \\
\hline
\end{tabular}




\section{REFERENCES}

Aurnague, M. (1995). Orientation in French spatial prepositions: Formal representations and influences. Journal of Semantics, 12, 239-267.

Bennett, D. C. (1975). Spatial and temporal uses of English: An essay in stratificational semantics. London: Longman.

Brugman, C. (1988). The story of 'over': Polysemy, semantics and the structure of the lexicon. New York: Garland Press.

Burgess, C., \& Lund, K. (1997). Modelling parsing constraints with high-dimensional space. Language and Cognitive Processes, 12, 177-210.

Carlson-Radvansky, L. A., Covey, E. S., \& Lattanzi, K. M. (1999). "What" effects on "where": Functional influences on spatial relations. Psychological Science, 10, $516-521$.

Carlson-Radvansky, L. A., \& Irwin, D. E. (1993). Frames of reference in vision and language: Where is above? Cognition, 46, 223-244.

Carlson-Radvansky, L. A., \& Irwin, D. E. (1994). Reference frame activation during spatial term assignment. Journal of Memory and Language, 33, 646-671.

Carlson-Radvansky, L. A., \& Logan, G. D. (1997). The influence of reference frame selection on spatial template construction. Journal of Memory and Language, 37, 411-437.

Carlson-Radvansky, L. A. \& Radvansky, G. A. (1996). The influence of functional relations on spatial term selection. Psychological Science, 7, 56-60.

Coventry, K. R. (1992). Spatial prepositions and functional Relations: The case for minimally specified lexical entries. Ph.D thesis, University of Edinburgh.

Coventry, K. R. (1998). Spatial prepositions, functional relations and lexical specification. In P. Olivier and K.-P. Gapp (Eds.), Representation and processing of spatial expressions (pp. 247-262). Mahwah, NJ: Erlbaum.

Coventry, K. R., Carmichael, R., \& Garrod, S. C. (1994). Spatial prepositions, object-specific function and task requirements. Journal of Semantics, 11, 289-309.

Coventry, K. R., \& Mather, G. (in press). The real story of over. In K. R. Coventry \& P. Olivier (Eds.), Spatial language: Cognitive and computational perspectives. Dordrecht: Kluwer Academic.

Garrod, S., C., Ferrier, G., \& Campbell, S. (1999). 'In' and 'on': Investigating the functional geometry of spatial prepositions. Cognition, 72, 167-189.

Garrod, S. C., \& Sanford, A. J. (1989). Discourse models as interfaces between language and the spatial world. Journal of Semantics, 6, 147-160.
Hayward, W. G., \& Tarr, M. J. (1995). Spatial language and spatial representation. Cognition, 55, 39-84.

Herskovits, A. (1986). Language and spatial cognition. An interdisciplinary study of the prepositions in English. Cambridge, UK: Cambridge Univ. Press.

Lakoff, G. (1987). Women, fire and dangerous things. Chicago: Chicago Univ. Press.

Landau, B., \& Jackendoff, R. (1993). "What" and "where" in spatial language and spatial cognition. Behavioral and Brain Sciences, 16, 217-265.

Landau, B., \& Munnich, E. (1998). The representation of space and spatial language: Challenges for cognitive science. In P. Olivier and K.-P. Gapp (Eds.), Representation and processing of spatial expressions (pp. 263272). Mahwah, NJ: Erlbaum.

Landauer, T., \& Dumais, S. (1997). A solution to Plato's problem: The latent semantic analysis theory of acquisition, induction and representation of knowledge. Psychological Review, 104, 211-240.

Levinson, S. C. (1996). Frames of reference and Molyneux's question: Crosslinguistic evidence. In P. Bloom, M. A. Peterson, L. Nadel, \& M. Garrett (Eds.), Language and space (pp. 109-169). Cambridge, MA: MIT Press.

Logan, G. D., \& Sadler, D. D. (1996). A computational analysis of the apprehension of spatial relations. In P. Bloom, M. A. Peterson, L. Nadel, \& M. Garrett (Eds.), Language and space (pp. 493-529). Cambridge, MA: MIT Press.

Regier, T. (1996). The human semantic potential. Spatial language and constrained connectionism. Cambridge, MA: MIT Press.

Sanford, A. J., \& Garrod, S. C. (1998). The role of scenario mapping in text comprehension. Discourse Processes, 26, 159-190.

Sedivy, J. C., Tanenhaus, M. K., Chambers, C. G., \& Carlson, G. N. (1999). Achieving incremental interpretation through contextual representation. Cognition, 71, 109147.

Talmy, L. (1983). How language structures space. In H. Pick $\&$ L. Acredolo (Eds.), Spatial orientation: Theory, research and application (pp. 225-282). New York: Plenum.

Talmy, L. (1988). Force dynamics in language and cognition. Cognitive Science, 12, 49-100.

Vandeloise, C. (1994). Methodology and analyses of the preposition 'in'. Cognitive Linguistics, 5, 157-184.

(Received September 25, 1998)

(Revision received June 1, 2000) 